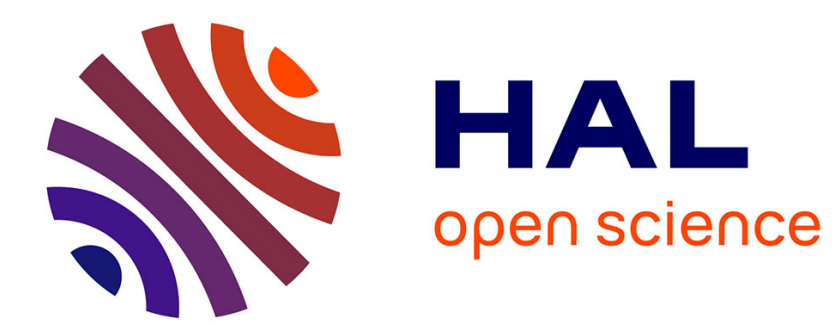

\title{
L'espace migratoire des Tunisiens en France
}

Gildas Simon

\section{- To cite this version:}

Gildas Simon. L'espace migratoire des Tunisiens en France. Espace Géographique, 1976, 2, pp.115120. hal-00609492

\section{HAL Id: hal-00609492 https://hal.science/hal-00609492}

Submitted on 19 Jul 2011

HAL is a multi-disciplinary open access archive for the deposit and dissemination of scientific research documents, whether they are published or not. The documents may come from teaching and research institutions in France or abroad, or from public or private research centers.
L'archive ouverte pluridisciplinaire HAL, est destinée au dépôt et à la diffusion de documents scientifiques de niveau recherche, publiés ou non, émanant des établissements d'enseignement et de recherche français ou étrangers, des laboratoires publics ou privés. 


\title{
L'ESPACE MIGRATOIRE DES TUNISIENS EN FRANCE
}

\author{
Gildas SIMON ${ }^{1}$
}

\begin{abstract}
RESUME. - Les Tunisiens résidant en France ont des structures de répartition et de migration différentes de celles des autres Maghrébins, avec lesquels ils sont souvent confondus. Ils se concentrent dans les plus grandes villes de l'axe Marseille-Lyon-Paris, et dans le secteur tertiaire. La pratique de leur espace migratoire suit des comportements et des schémas mentaux qui relèvent de la sociologie régionale tunisienne.

Comportement migratoire ; emploi ; France ; secteur tertiaire ; travailleurs étrangers ; Tunisie

ABSTRACT. - The settlement patterns of Tunisians in France. Tunisians who live in France have different social and settlement structures from other Maghrebians. They gather in the largest towns in the Marseilles-Lyons-Paris axis and work mainly in the tertiary sector. Their settlement pattern is linked to behavioural and mental pro cesses which reflect the regional sociology of Tunisia.
\end{abstract}

Employment ; France ; guest workers ; migration behavior ; tertiary sector ; Tunisia

L'analyse des migrations de travailleurs gagne à être menée à la fois au lieu de départ et au lieu d'arrivée : il y a des rapports intéressants entre la nature des relations sociales dans la région de départ, les filières migratoires et les orientations spatiales ou professionnelles à l'arrivée. Le cas des Tunisiens en France est assez original, même par rapport à l'ensemble de l'immigration maghrébine, pour être isolé.

\section{La spécificité de l'espace migratoire des Tunisiens en France.}

Selon les statistiques du Ministère de l'Intérieur, valables au 31 décembre 1973, on peut estimer le nombre des Tunisiens en France à 161 000, y compris la population familiale de la Région parisienne. Le chiffre actuel présente l'avantage d'intégrer un bon nombre de travailleurs entrés en France en qualité de «touristes» depuis plusieurs années, et dont la situation administrative a été régularisée exceptionnellement au cours de l'été 1973 : 16883 Tunisiens sur une entrée annuelle de 22000 sont dans ce cas.

Mais il est possible que le chiffre global donné ici, ne pouvant tenir compte des retours définitifs en Tunisie, soit l'objet d'une certaine surestimation à cause du pourcentage élevé des

\footnotetext{
${ }^{1}$ Professeur de géographie, MIGRINTER - Université de Poitiers / CNRS : UMR 6588
} 
titres de séjour de durée supérieure à 1 an. Ces statistiques ont servi de base à l'établissement de la fig. 1 et permis de suivre l'évolution à intervalles presque réguliers (1958-1966-1973).

\section{a. Une immigration récente et concentrée.}

La population tunisienne en France est nettement plus concentrée que l'ensemble de la population étrangère puisque, en 1973, 80 \% des Tunisiens résident dans trois régions seulement (Région parisienne, Rhône - Alpes, Provence - Côte-d'Azur - Corse) alors que ce taux est de $57 \%$ pour toute la population étrangère. Un tel regroupement est l'expression d'une immigration relativement récente et dotée d'une grande cohésion sociale.

A la différence de la migration algérienne et marocaine, nettement plus ancienne, l'immigration tunisienne ne débute qu'en 1956, lors de l'indépendance de la Tunisie, et elle ne prend véritablement son essor qu'en 1965-66. Jusqu'à une date récente, elle se cantonne dans ses premiers points d'appui et n'affecte pas l'ensemble du territoire français, où d'autres groupes nationaux sont, par contre, solidement implantés depuis une ou plusieurs générations.

La force de ses structures sociales, un sens familial et communautaire relativement développé, jouent un rôle important dans la concentration de cette population. On sait l'influence de ces liens pour la recherche d'un emploi et d'un logement. De 1967 à 1973, 90 \% des travailleurs permanents 'contrôlés par l'Office National d'Immigration (ONI) ont été soit régularisés sur place, soit introduits sur contrats nominatifs obtenus par les intéressés eux-mêmes ou par relations familiales ou sociales; le groupement de ces travailleurs dans les trois principales régions est resté supérieur à $80 \%$. La seule année où ce taux a enregistré une baisse notable (67 \%) est 1969 où, par suite de la mise en application de l'accord de main-d'oeuvre entre les deux Etats, la Tunisie a bénéficié d'un nombre important de contrats anonymes, ce qui a déterminé une certaine dispersion de la main-d'oeuvre recrutée. On voit ainsi la faiblesse des facteurs administratifs dans la répartition de cette population, par rapport à la force des contraintes sociologiques ou des agents économiques.

\section{b. L'axe Paris-Méditerranée et le relais lyonnais.}

Selon la fig. 1, l'espace migratoire des Tunisiens en France correspond au dispositif classique de l'immigration maghrébine en s'appuyant sur un axe principal Marseille-Lyon-Paris et sur un axe secondaire Marseille-Toulon-Nice. Cette disposition met en valeur le rôle charnière de Marseille qui sert de porte d'entrée plutôt que de point de fixation, et le rôle de relais exercé par Lyon. Ce dernier apparaît sur les fig. 2 et 3, établies selon les déclarations de changement de résidence effectuées par les intéressés en 1973 auprès des préfectures des lieux d'arrivée et retransmises ensuite à la Préfecture du Rhône. Outre les relations de cette métropole avec son espace régional (migrations importantes avec la Loire, l'Ain, l'Isère, la Savoie), on y voit également la solidarité géographique qui existe à l'intérieur de cet espace migratoire entre Paris, Lyon et la côte méditerranéenne.

Il faut remarquer toutefois que les Tunisiens n'utilisent pas la totalité de l'espace maghrébin en France, dans la mesure où leur représentation en Lorraine et dans le Nord est particulièrement faible. Dans le Nord, par exemple, il n'y avait en 1971 que 2,1 \% de leurs effectifs, contre 9,8 \% des Marocains : les Tunisiens sont peu nombreux dans les régions de mines et d'industries lourdes, car ils sont faiblement attirés par ce type d'emplois, occupés par les Marocains et par les Algériens depuis une ou plusieurs dizaines d'années. La relève de ceux-ci s'effectue aujourd'hui par les Turcs, du moins en Lorraine. 


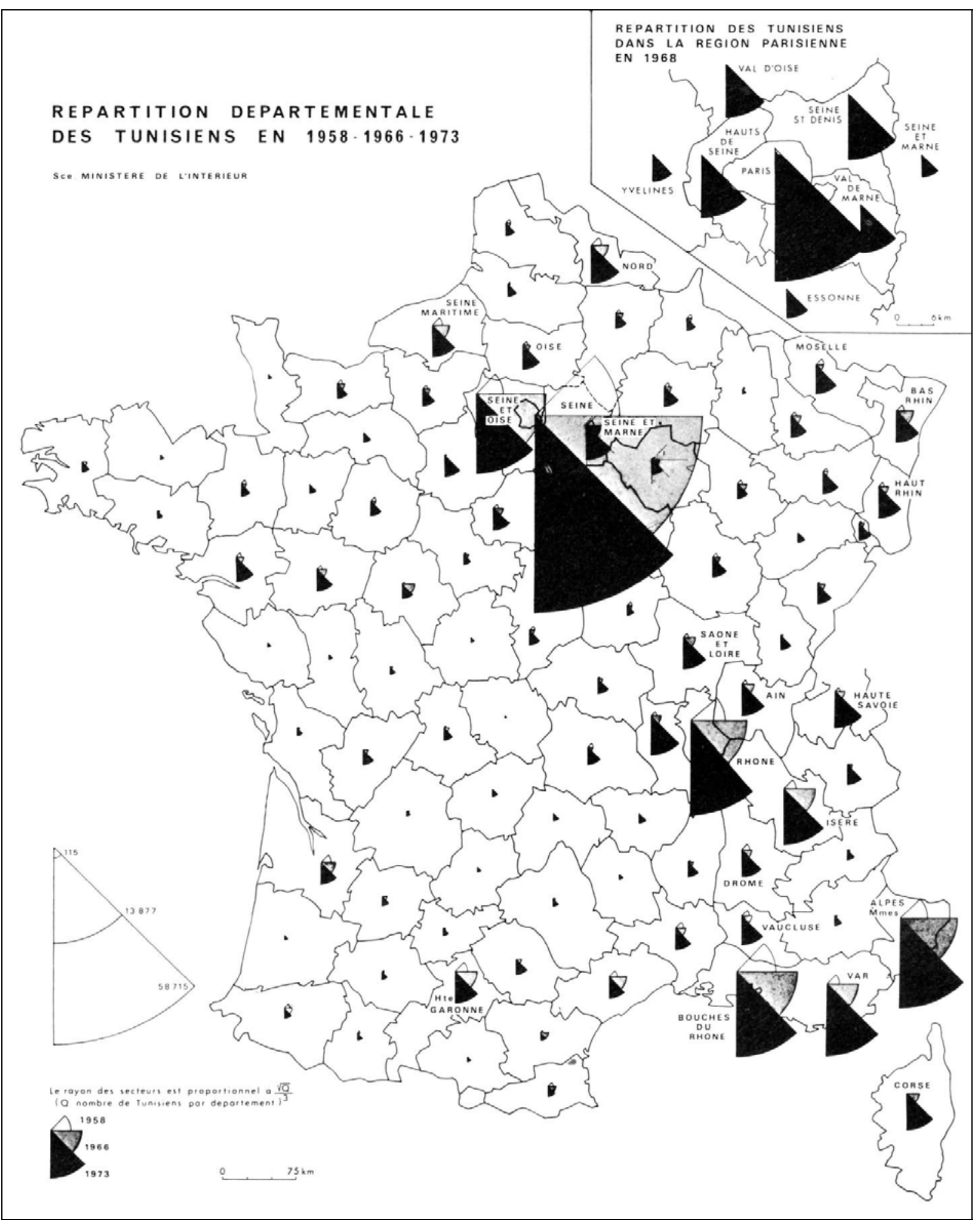

FIG. 1. - Répartition des Tunisiens en France.

La surface des secteurs est proportionnelle aux nombres de Tunisiens par département (Paris en 1973: 58715).

- Noir: 1973.

- Gris: 1966.

- Blanc: 1958. 


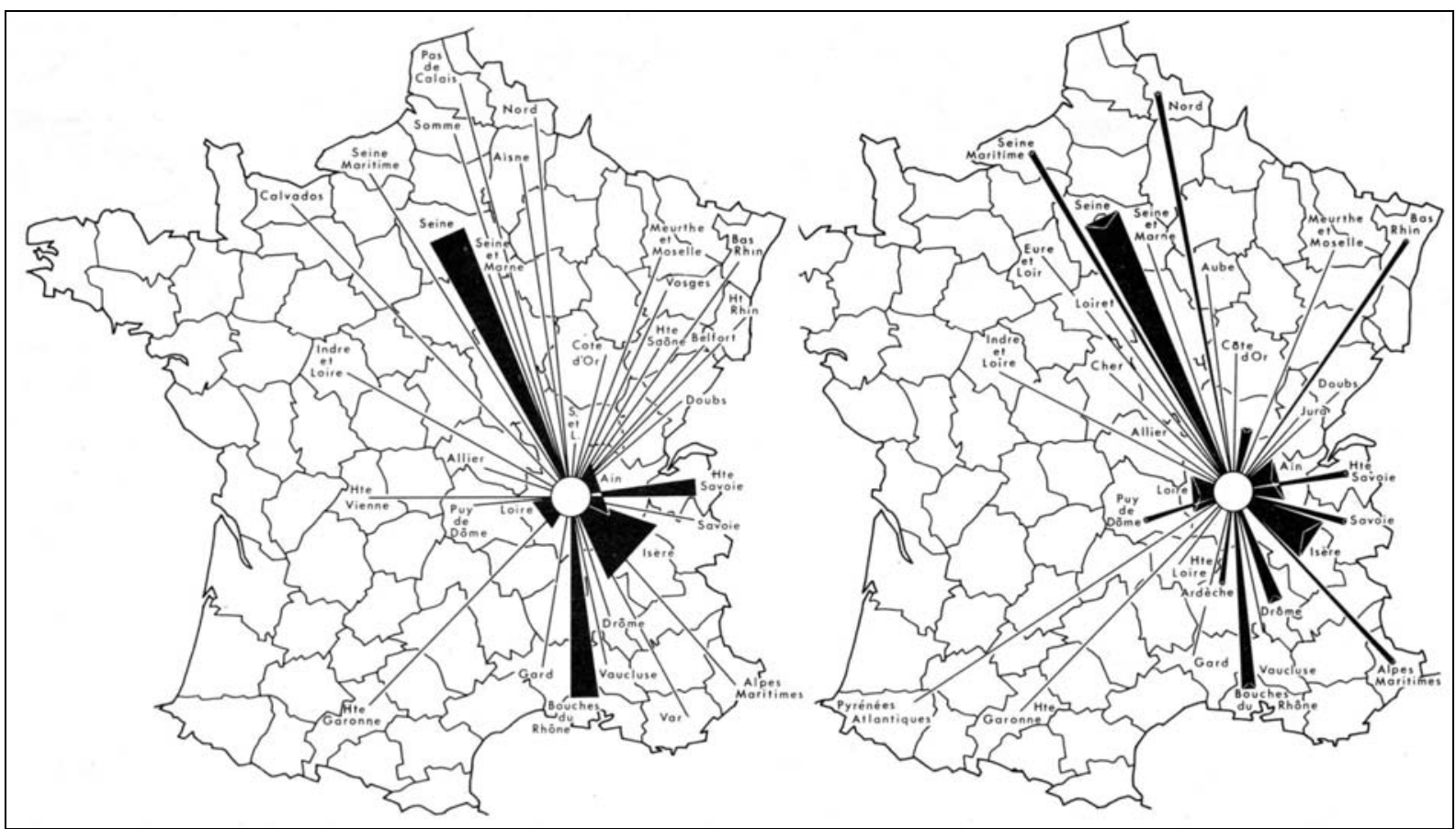

FIG. 2 et 3. - Migrations internes des Tunisiens au départ de Lyon (à gauche) et vers Lyon (à droite) en 1973.

De même, le peu d'intérêt pour les activités agricoles explique leur rareté dans les zones rurales, parce que leurs aptitudes et leurs préférences professionnelles les poussent à la recherche des professions spécifiquement urbaines, quitte à accepter un premier emploi dans l'agriculture, le bâtiment ou les travaux publics.

\section{c. L'intense attraction des grandes agglomérations urbaines.}

Dans une étude précédente, réalisée en collaboration avec D. Noin ${ }^{2}$ (1), on avait noté l'attrait considérable des grandes villes sur les Maghrébins en général et sur les Tunisiens en particulier. Selon le recensement de 1968, 75 \% des Marocains, 78 \% des Algériens et $89 \%$ des Tunisiens résidaient dans des agglomérations supérieures à 50000 habitants. Paris l'emportait nettement avec $54 \%$ des Marocains, $37 \%$ des Algériens et $62 \%$ des Tunisiens.

Une telle concentration dans les grandes villes est due selon nous à deux raisons : la nature des emplois recherchés, sinon obtenus; l'attirance du milieu urbain, compte tenu de l'origine géographique des migrants et du degré d'urbanisation de la Tunisie.

Les statistiques de l'ONI et la publication récente d'une enquête du Ministère du Travail sur la main-d'œuvre étrangère dans les établissements industriels et commerciaux en 1971 permettent de déterminer le type d'insertion professionnelle des Tunisiens en France et d'en dégager les implications spatiales (tableaux 1 et 2).

Le trait le plus notable est leur importance dans le bâtiment, et dans les activités du secteur tertiaire très caractéristiques du milieu urbain : les transports, les services et surtout le commerce. Le même type de relations emploi-localisation vaut également pour la localisation à l'intérieur des villes. Dans l'agglomération parisienne, les Tunisiens sont particulièrement regroupés dans le quart nord-est de la ville (XVIIIe, XIxe, XXe et XIe arrondissements) alors

${ }^{2}$ G. SIMON et D. NOIN, La migration maghrébine vers l'Europe. Cahiers d'Outre-Mer, 1972. 
que les Marocains, plus portés vers les activités industrielles, occupent les banlieues ouvrières du nord (Seine-Saint-Denis) et surtout du nord-ouest (Hauts-de-Seine, à Gennevilliers notamment). La force du regroupement sociologique est telle qu'elle peut dépasser la liaison emploi-localisation. Ainsi, la grande majorité des ouvriers tunisiens employés aux établissements Renault de Boulogne-Billancourt résident dans le quartier nord-est de Paris et non dans la banlieue nord-ouest comme on aurait pu l'imaginer.

TABLEAU 1

Admission des travailleurs permanents sur le marché de l'emploi (en \%, 1967-1973)

\begin{tabular}{|l|c|c|c|}
\hline & Tunisiens & Marocains & Etrangers \\
\hline Agriculture & 9,9 & 21,8 & 12,0 \\
\hline Bâtiment et T.P. & 44,3 & 27,2 & 33,7 \\
\hline Industrie & 24,9 & 37,8 & 33,4 \\
\hline $\begin{array}{l}\text { Transports, services et } \\
\text { commerces }\end{array}$ & 20,9 & 13,2 & 22,9 \\
\hline TOTAL & 100,0 & 100,0 & 100,0 \\
\hline
\end{tabular}

Source : ONI

TABLEAU 2

Répartition des salariés tunisiens, marocains et étrangers dans les établissements industriels et commerciaux au $1^{\mathrm{er}}$ juillet 1971

\begin{tabular}{|l|c|c|c|}
\hline & Tunisiens & Marocains & Etrangers \\
\hline Bâtiment et T.P. & 41,9 & 32,4 & 38,4 \\
\hline Industries & 40,0 & 59,6 & 50,6 \\
\hline $\begin{array}{l}\text { Transports, services et } \\
\text { commerces }\end{array}$ & 18,1 & 8,0 & 11,0 \\
\hline TOTAL & 100,0 & 100,0 & 100,0 \\
\hline
\end{tabular}

Source: Ministère du Travail. Etablissements de plus de 10 salariés, à l'exclusion de l'agriculture et des entreprises nationalisées.

\section{d. L'élargissement du champ migratoire.}

Cependant, cet espace migratoire fortement polarisé connaît une nette évolution depuis 1966. On constate sur la fig. 1 un double mouvement de déconcentration au profit des espaces périphériques des grandes villes et des autres régions. L'exemple du desserrement qui s'effectue autour de Lyon est le plus typique. De 1966 à 1973, la croissance de la population tunisienne a été nettement plus forte dans les départements périphériques (Loire, Saône-etLoire, Ain, Isère et Savoie) que dans le département du Rhône. Sur le plan national, on peut observer également une implantation relativement récente dans des régions très extérieures à l'axe principal, en Alsace ou dans les Pays-de-la-Loire (Maine-et-Loire et surtout LoireAtlantique). On peut attribuer cette tendance aux difficultés du marché de l'emploi dans les lieux d'arrivée traditionnels et à l'expansion économique des autres régions. Mais elle est peutêtre aussi l'expression d'un enracinement plus solide en France et d'une certaine assurance permettant la prospection de l'ensemble du territoire.

\section{Le partage sociologique de l'espace migratoire.}

L'étude de l'origine régionale des Tunisiens et de leur distribution dans le cadre spatial précédemment défini montre que leur répartition n'y est pas uniforme, mais répond à des critères d'ordre sociogéographique. La base de ,cette recherche repose sur des enquêtes directes dans les villages de Tunisie et sur des dépouillements statistiques de sources françaises et tunisiennes - notamment l'origine des mandats postaux émis en France et payés en Tunisie. Cette dernière source a servi de base à l'établissement de la fig. 4 . 


\section{a. La sélection régionale par les groupes sociogéographiques.}

On retrouve sur cette carte les ensembles régionaux : la région tunisoise, le Cap Bon, le Sahel de Sousse et de Sfax, le Tell intérieur, les Steppes centrales, le Sud côtier et intérieur. Chaque groupe régional possède en France ses propres structures de répartition, et utilise l'espace migratoire selon des schémas qui lui sont propres. Une véritable sélection régionale s'effectue au départ, par l'intermédiaire des filières migratoires.

Une opposition fondamentale se manifeste entre la zone côtière orientale, qui est fortement polarisée par la Région parisienne, et l'intérieur, dont les migrants sont attirés vers les autres régions. Selon le fichier des titres de séjour à Paris, 87 \% des Tunisiens installés dans Paris et la proche banlieue proviennent des gouvernorats de la côte orientale, dont $21 \%$ de Tunis : cette distindion fait apparaître une fois de plus un contraste fondamental de la géographie humaine tunisienne. Cette attraction de la Région parisienne nous parait en rapport avec l'urbanisation plus forte de cet ensemble par rapport à l'intérieur. Le littoral groupe en effet les villes les plus importantes (Tunis, Sousse, Sfax) et les plus dynamiques du pays, connaît les taux d'urbanisation les plus élevés. Cette constatation s'applique également à la capitale, surtout à la Tunisie urbanisée qui s'étend jusqu'à: la bordure côtière du Sud, de Gabès à Djerba et à Tataouine, zone polarisée depuis longtemps par Tunis, ainsi que le prouvent l'importance et la direction des flux migratoires internes ${ }^{3}$. Les migrants de cette zone côtière rechercheraient à Paris un milieu sociologique et professionnel relativement conforme à leur milieu d'origine. Il est intéressant de voir le même phénomène dans l'émigration israélite, car près de $80 \%$ des Juifs installés définitivement à Paris viennent de Tunis, $15 \%$ de Sousse et Sfax. Ils exercent dans la capitale française des activités artisanales (confection de vêtement) ou tertiaires (professions libérales et commerciales) très comparables à celles exercées précédemment dans les villes, dont ils sont originaires ${ }^{4}$.

Les migrants de l'intérieur se dirigent vers trois autres types de régions: les montagnards du Tell intérieur vont surtout sur les côtes méditerranéennes françaises à l'est de Marseille (Bouches-du-Rhône, Alpes-Maritimes, Var et Corse), soit dans le bâtiment, soit dans les activités agricoles (Corse, Alpes-Maritimes, Vaucluse); les habitants du Sud intérieur (Djebalias, Nefzaouas) sont attirés par Lyon où ils travaillent dans le bâtiment et dans l'industrie; les migrants de la zone steppique se dispersent; ceux qui viennent des villes (Kairouan) ou des noyaux en cours d'urbanisation (Beja, Jendouba, Gafsa) vont, comme les citadins du littoral, à Paris.

\section{b. Les facteurs sociologiques de regroupement ou de dispersion en France.}

L'accumulation dans la même région d'arrivée, voire dans le même département ou la même ville, démontre à l'évidence le rôle essentiel joué par les structures sociologiques de départ. De leur force ou de leur faiblesse dépendent la présence ou l'absence de filières migratoires assurant au migrant la sécurité collective du groupe. Le degré de cohésion renforce la sélection et la concentration régionale en France.

\footnotetext{
${ }^{3}$ GROUPE HUIT, Villes et développement en Tunisie. Tunis, 1973.

${ }^{4}$ G. SIMON, Musulmans et Israélites tunisiens dans l'agglomération parisienne. Population, 1974, ${ }^{\circ}$ spécial Migrations.
} 


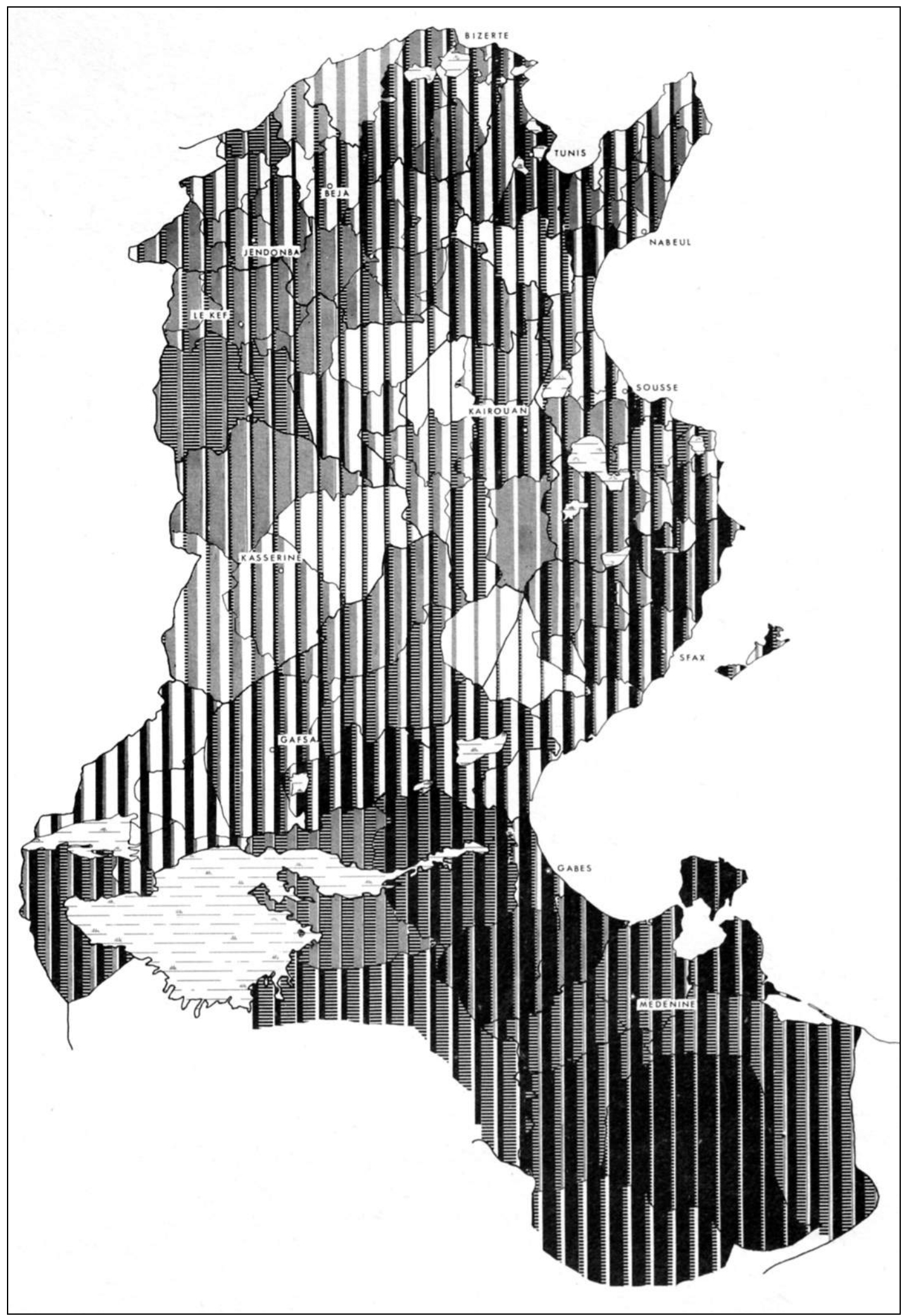

FIG. 4. - Attraction des régions françaises sur l'émigration tunisienne.

- Noir: Région parisienne. - Gris: Provence-Côte d'Azur et Corse.

- Hachures horizontales: Rhône-Alpes. - Blanc: Autres régions.

Source: Mandats postaux de Tunis.

Les groupes à forte cohésion sociale appartiennent généralement à des communautés rurales de paysans sédentaires, fortement attachés à la région ou au village dont ils sont originaires : 
Djebalias des Monts de Matmata et de Tataouine, habitants des oasis continentales (Nefzaouas) ou côtières (Mareth, Djerba).

Le cas de Djerba est l'un des plus caractéristiques. Selon les contrats de travail et les mandats postaux envoyés dans l'île en 1973, 85 \% de ses migrants résident dans la Région parisienne, $7 \%$ dans la Région lyonnaise, $2 \%$ dans le Midi et $6 \%$ ailleurs ; $83 \%$ des « Parisiens» se retrouvent à Paris intra-muros et plus particulièrement dans les IIIe, Ve, XIe, XVe et XVIIIe arrondissements. Selon les contrals de travail, $90 \%$ de leurs emplois concernent le secteur tertiaire : l'hôtellerie, la restauration et les services de nettoiement. Une petite minorité seulement se livre au commerce de détail, comme en Tunisie. On retrouve le même schéma de répartition dans les oasis voisines de Zarzis, mais avec un taux de concentration légèrement moins accusé: 70 \% dans la Région parisienne, $15 \%$ dans la Région lyonnaise, 2 \% dans le Midi let $13 \%$ ailleurs. La différence entre les deux foyers voisins pourrait s'expliquer par le caractère moins homogène de la population, provenant des délégations de l'intérieur du gouvernorat de Médenine. Certaines communautés rurales du Tell intérieur présentent également un taux de regroupement très fort, soit dans la région méditerranéenne (à Thala 71 \% des migrants, à Sakiet Sidi Youssef 65 \%) soit dans la Région lyonnaise (à Tadjerouine 67 $\%)$.

D'autres groupes, également très structurés, ont deux points d'appui distincts. Tel est le cas des travailleurs de Kebili, dans le Sud intérieur, dont 50 \% résident dans la Région RhôneAlpes (essentiellement à Lyon et à Saint-Etienne) et $39 \%$ à Nice ou dans les environs immédiats, où ils sont souvent employés dans l'horticulture, mettant ainsi à profit la réputation d'excellents jardiniers dont ils jouissent en Tunisie.

Les groupes déstructurés. - A ce premier type de comportement migratoire s'oppose celui des migrants appartenant à des groupes déstructurés, que la déstructuration sociale soit l'effet de la sédentarisation dans les régions d'élevage semi-nomade, la conséquence de l'exode rural ou de la scolarisation. Ces émigrés ont un comportement de type individualiste et se dispersent dans tout l'espace migratoire.

TABLEAU 3

Corrélations entre les migrations internes vers Tunis (1966) et les émigrations vers la France (1970)

\begin{tabular}{|l|c|c|}
\hline & $\begin{array}{c}\text { \% migrants vers } \\
\text { Tunis 1966 (1) }\end{array}$ & $\begin{array}{c}\text { \% migrants vers la } \\
\text { France1970 (2) }\end{array}$ \\
\hline Le Kef & 13,5 & 9,0 \\
\hline Jendouba & 7,1 & 8,8 \\
\hline Beja & 15,7 & 4,0 \\
\hline Bizerte & 9,7 & 12,3 \\
\hline Nabeul & 7,6 & 6,1 \\
\hline Sousse & 12,0 & 21,8 \\
\hline Sfax & 6,8 & 9,7 \\
\hline Kairouan & 4,5 & 3,0 \\
\hline Kasserine & 3,8 & 3,0 \\
\hline Gafsa & 3,1 & 2,1 \\
\hline Gabès & 6,6 & 7,7 \\
\hline Médenine & 9,6 & 12,5 \\
\hline
\end{tabular}

$T=0,458$; $T$ sans Beja : 0,775.

Sources : INS Tunis (1) et ON! Paris (2).

Tel est le cas de ceux qui viennent de la Tunisie steppique et des grandes plaines céréalières du Nord. Les migrants, qui partent seuls ou en couple, paraissent particulièrement mobiles en 
France et ont plus de difficultés que les autres à s'adapter aux difficiles conditions de vie de la migration. Corrélativement, il n'y a pas de véritable filière migratoire. La carte de la dispersion en France coïncide avec celle des flux les plus faibles.

Les migrants d'origine urbaine, plus individualistes, présentent aussi une certaine tendance à: la dispersion, mais masquée par une concentration notable dans l'agglomération parisienne : selon les mandats, $32 \%$ des originaires de Sousse, $45 \%$ de Sfax, $47 \%$ de Tunis, $55 \%$ de Gabès. Ce regroupement de fait s'expliquerait plus par l'attraction sociologique du milieu d'arrivée que par la cohésion du groupe migrant.

\section{L'espace migratoire externe est-il une projection de l'espace migratoire interne?}

Hormis les raisons évoquées ci-dessus, on peut se demander si l'espace migratoire des Tunisiens en France n'est pas également l'expression d'une certaine représentation mentale de l'espace, dont le schéma serait celui de l'espace migratoire interne, en Tunisie.

La comparaison de cartes de migrations internes et d'émigrations montre une certaine concordance d'ensemble. Les régions à fort coefficient migratoire interne connaissent également des flux externes importants. Tel est le cas des montagnes du Tell intérieur, du Sahel de Sousse et surtout du Sud. On note par, contre la faiblesse des départs dans le Cap Bon, le Sahel sfaxien et, surtout, dans toute la Tunisie centrale. Il y a bien des distorsions : tel village du Sud, Matmata, n'envoie ses migrants qu'en Tunisie, alors que l'oasis voisine de Mareth est entièrement polarisée par la France; mais elles sont locales et ne semblent pas entamer la concordance du schéma général.

D'autre part, il est intéressant de constater la similitude d'attraction des deux capitales dans l'espace migratoire: Tunis domine les migrations intra-tunisiennes (46,6 \% des migrants), comme Paris les migrations vers la France. Simple manifestation de la concentration métropolitaine ? En fait, il existe aussi une coïncidence assez nette entre la répartition régionale de migrants vers Tunis en 1966 et le taux de migrants vers la France en 1970 (Tunis exceptée). Une seule exception : le gouvernorat de Beja, qui appartient à la zone déstructurée sur le plan sociologique.

Cette similitude peut s'expliquer par une réaction identique de départ face aux difficultés des économies régionales, mais elle s'interprète peut-être aussi par le fait que l'attraction de la capitale tunisoise et celle de l'étranger sont perçues par la population de manière comparable. Il y aurait là un champ de recherche intéressant à approfondir dans le domaine psycho-social. A titre d'hypothèse, certaines motivations apparaissent communes: augmentation du nombre de chances de trouver un emploi; attrait de l' « urbanité » et de la " modernité » que peuvent représenter également la capitale tunisienne et l'émigration en France. L'espace où ,ces motivations peuvent être ressenties avec le plus de force sont sous l'influence de Tunis, ce qui pose le problème plus vaste des rapports entre l'émigration et la polarisation de l'espace tunisien par la capitale tunisoise.

Ce problème est essentiel pour comprendre la géographie dynamique de la population tunisienne actuelle. On peut se demander si l'expansion de l'espace migratoire externe, qui s'est élargi à la France entière et aussi à l'Allemagne et à la Libye, ne va pas remettre en cause les structures de l'espace migratoire interne. 